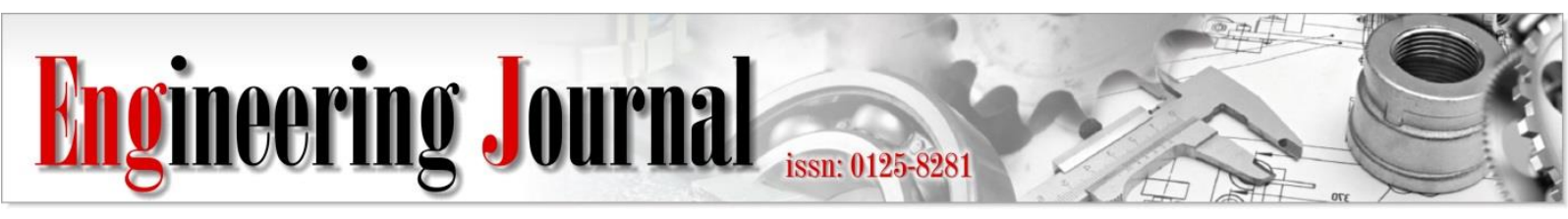

Article

\title{
Efficiency on Fungal Inhibition of Benzalkonium Chloride and Lemongrass Essential Oil on Polyester Fabric
}

\author{
Thanyanuch Kriangkraipipat ${ }^{1, a}$, Nattawat Wittayakunsathit ${ }^{2, b}$, Thawat Ngamsritrakul ${ }^{2, c}$, \\ and Sirima Panyamethekul ${ }^{2, \mathrm{~d}, *}$
}

1 Department of Microbiology, Faculty of Science, Chulalongkorn University, Bangkok 10330, Thailand 2 Department of Environmental Engineering, Faculty of Engineering, Chulalongkorn University, Bangkok 10330, Thailand

E-mail: athanyanuch.k@chula.ac.th, bthe_rock0868@hotmail.com, ckim.ngamsritrakul@gmail.com, dSirima.P@Chula.ac.th (Corresponding author)

\begin{abstract}
These days, people spend most of their time indoors as a result indoor air quality is a great concern. Mold growth in indoor environment may significantly contribute to unhealthy air quality and to deterioration of building materials. Upholstery fabrics are made of polyester fabrics, on which fungi can grow. Besides, fungi naturally grow in a high humidity condition, seem expected in dwellings and buildings with limit ventilation. This study applied Benzalkonium Chloride and lemongrass essential oil to inhibit the fungal growth on fabrics. Aspergillus niger, a prevalent airborne fungus, was tested on polyester fabrics including, TC fabric ( $65 \%$ polyester $/ 35 \%$ cotton), and TK fabric ( $100 \%$ polyester), according to AATCC Test Method 20-2008, 30-2004 Section III, and 81-2006. The results indicated that BKC was more applicable than lemongrass essential oil regarding minimum inhibitory concentration and cost of fungicides. One percent and 5\% BKC are suitable to inhibit the fungal growth on TC and TK fabrics, respectively. Effective duration of $1 \% \mathrm{BKC}$ and $5 \% \mathrm{BKC}$ are 18 and 30 days, respectively. Furthermore, both fabrics were not damaged by $1 \%$ BKC and $5 \%$ BKC when inspected under the microscope. In conclusion, $\mathrm{BKC}$ would be recommended for inhibiting fungal growth on polyester fabrics.
\end{abstract}

Keywords: Polyester fabric, antifungal agent, fungi, indoor air quality.

ENGINEERING JOURNAL Volume 24 Issue 1

Received 26 June 2018

Accepted 4 December 2019

Published 8 February 2020

Online at http://www.engj.org/

DOI:10.4186/ej.2020.24.1.23 


\section{Introduction}

Lifestyle of people in the big city are dominate by indoor activities. Urban residents may spend most of their time indoors up to more than 21 hour per day. Indoor environmental quality is afterward essential. Dampness present in buildings in hot-humid climates is a limiting factor for fungal growth. Fungi naturally grow in suitable conditions such as temperature between $25-30{ }^{\circ} \mathrm{C}$, high relative humidity (over 60\%), and $\mathrm{pH}$ range from 5.0-6.0. Organic materials such as leaves, woods, and papers are main food of fungi for growth and reproduction. Fungi would damage construction materials, furniture, fixtures and cause negative health effect [1-2]. The most common indoor fungi are Cladosporium, Penicillium, Aspergillus, and Alternatia [3]. Fungi could change the material surface colors to their spore colors. For example, black mold like Aspergillus niger could cover material surface and then change the surface into black. In addition, fungi are commonly found in air-conditioned environment [4-5]. They spread into ambient and would cause material surface damage and health effect such as allergy, asthma, and tinea [6]. Highrise and large buildings with A/C system including, apartment, office, cinema and department store, are examples of indoor settings being full of people at a time. Upholstery fabrics are usually made of polyester fabric because of its economical price. But polyester fabric is an organic material that is a main food of fungi [7]. The study, then seeks proper solutions to prevent mold growth on polyester fabric.

The general antifungal agents are chemical products; sodium hypochlorite $(\mathrm{NaOCl})$, and hydrogen peroxide, for instance. These antifungal agents could cause adverse health effect [8], such as coughing, sore throat, irritation, and headache. Benzalkonium chloride (BKC) is a quaternary ammonium compound (QAC) being called "green" solvents and biodegradable [9-10]. It is nontoxic for homeothermic organisms. BKC at a concentration of $0.1 \%$ is used as a preservative in eye drops [11]. Low concentration of BKC in a range of $8-32 \mu \mathrm{g} / \mathrm{mL}$ had been reported to inhibit growth of Fusarium spp., Aspergillus spp., and Alternaria spp. [12]. BKC will be explored as a synthetic fungicide in this study. Lemongrass essential oil is a natural fungicide extracted from a plant, Cymbopogon citratus (DC.) Stapf, commonly known as lemon grass. The oil mostly composed of monoterpene compounds, geranial $(42.2 \%)$, and neral $(31.5 \%)$ [13]. It has been shown to be effective against various fungi causing postharvest spoilage on vegetables and fruits at 25-500 ppm [14].

This research aims to seek minimum inhibitory concentration (MIC) and effective duration of both fungicides on polyester fabrics. The damage of polyester fabrics caused by both fungicides is also evaluated in this study.

\section{Materials and Methods}

\subsection{Antifungal Agents}

Benzalkonium chloride (BKC) and lemongrass essential oil (Chemipan corp., Bangkok, Thailand) were tested in this study. BKC were prepared at concentration of $1 \%, 5 \%, 10 \%, 15 \%, 20 \%$, and $25 \%$. Lemongrass essential oil was prepared at concentration of $1 \%, 2 \%, 3 \%$, $4 \%, 5 \%$, and $10 \%$. Fifty percent ethanol was used as a solvent of lemongrass essential oil in this study.

\subsection{Determination of $\mathrm{pH}$ of Polyester Fabric}

Acidic or alkaline chemical residues left from textile production and finishing may deviate $\mathrm{pH}$ of the fabric from habitable range of fungi. To ensure a quantitative determination of antifungal agents, fabric was tested according to American Association of Textile Chemists and Colorists (AATCC) Test Method 812006. Five replicates of each fabric were done. The $\mathrm{pH}$ of the fabric is defined as the $\mathrm{pH}$ of the water present in the extract.

\subsection{Test Culture and Media}

Aspergillus niger, American Type Culture Collection No. 6275 or TISTR \#3245, was used as fungi delegate. The media in this study was Mineral salts agar [15].

\subsection{Fungal Inhibition Test}

The test was performed according to AATCC Test Method 30-2004 Section III. Aspergillus niger was cultured on potato dextrose agar medium and then incubated at 30 ${ }^{\circ} \mathrm{C}$ for 7 days. Sterile water was added to the plate to make a spore suspension of the fungus. A volume of $0.4 \mathrm{ml}$ of freshly prepared conidia $\left(10^{5}\right.$ spores $\left./ \mathrm{ml}\right)$ was spread on the Mineral salts agar plate. The polyester fabric TC $(65 \%$ polyester $/ 35 \%$ cotton) and TK (100\% polyester) were cut into $3.8 \pm 0.5 \mathrm{~cm}$ diameter. The specimens were damped with BKC and lemongrass essential oil. Then, the fabric was put on the center of the plate. All the specimens were incubated at $30^{\circ} \mathrm{C}$ for 14 days. Five replicates were done in this study. Evaluation of mold growth is as follows [15]:

- No fungal growth (report the clear zone in $\mathrm{mm}$ )

- Microscopic fungal growth (visible only under the microscope)

- Macroscopic fungal growth (visible to the eye)

\subsection{Effective Duration Test}

The experiment was set up as previously described in the fungal inhibition test (2.3) with up to 30 days of incubation. Fungal occurrence was observed on day 7, 15, and 30. Effective duration of fungicide was according to the date of fungal occurrence on TC and TK. If fungus was occurred on TC and TK, the previous observed date 
would be the effective duration of fungicide. For example, fungus on TC was occurred on date 30 , the effective duration of fungicide on TC was then 15 days. Five replicates were done for each experiment.

\subsection{Damage of Polyester Fabric Caused by Fungicide Test}

TC and TK fabric were cut into $3.8 \pm 0.5 \mathrm{~cm}$ diameter. The specimens were damped with $1 \%, 5 \%, 10 \%, 15 \%$, $20 \%, 25 \%$, and $80 \%$ BKC concentrations and $1 \%, 2 \%$, $3 \%, 4 \%, 5 \%, 10 \%$, and $100 \%$ lemongrass essential oil. In control sets, the specimens were damped with distilled water (DI) or $50 \%$ ethanol that were diluents of fungicides. These specimens were put on the center of petri dish. The damage of polyester fabric caused by fungicide was analyzed by lost weight and characteristic of fiber arrangement. The lost weight of TC and TK were measured on a 4-digits weighing apparatus. The characteristic of fiber arrangement was examined under USB digital microscope according to AATCC Test Method 20-2008. The analytic periods were on day 1, 3, 7 , 10, and 14 . Three replicates were done.

\section{Results and Discussion}

\section{1. $\mathrm{pH}$ of the TC and TK Fabrics}

The range of $\mathrm{pH}$ supporting growth of mold is quite broad, $\mathrm{pH} 3.0-8.0$, with optimum around $\mathrm{pH}$ 5.0. Optimal $\mathrm{pH}$ for growth of $A$. niger were between 4 and 6.5 [16]. The $\mathrm{pH}$ of the water-extract from the TC fabric was $5.61 \pm 0.0057$, while the $\mathrm{pH}$ of the water-extract from TK fabric was $6.75 \pm 0.0058$. Both were in the growable range of the fungus. The results suggested that $\mathrm{pH}$ of the fabrics was not a factor inhibiting growth of $A$. niger.

\subsection{Efficiency on Fungal Inhibition of Both Antifungal Agents}

3.2.1. Efficiency on fungal inhibition of $B K C$ and lemongrass essential oil on TK fabric

After 14 days of incubation, the mold inhibition was evaluated by observing fungal growth according to Test Method 30-2004 Section III. TK fabrics with higher BKC concentrations had a bigger clear zone as shown in Fig. 1. One percent BKC, Fig. 1(a), has the lowest clear zone of $46 \mathrm{~mm}$. The 5\% BKC, Fig. 1(b), 10\% BKC, Fig. 1(c), 15\% BKC, Fig. 1(d), and 20\% BKC, Fig. 1(e), had a 56, 77, 74, and $78 \mathrm{~mm}$ of clear zone, respectively. And the widest clear zone of mold growth was on 25\% BKC, Fig. 1(f). Figure 2 depicted the correlation between clear zones of fungal inhibition on TK fabric and BKC concentrations. As seen in Fig. 2, the clear zone was enhanced as the BKC concentrations increase. All BKC concentration tested, $5 \%-25 \%$, could inhibit fungal growth on $40 \mathrm{~mm}$ diameter discs of TK fabric. One percent BKC was the MIC of TK fabric in this study.

Ethanol was used as a solvent for lemongrass essential oil dilution in this study. The alcohol effect on efficiency of fungal inhibition was then considered. Figure 3 showed that all concentrations exploited $(1 \%-50 \%)$ did not influence an antifungal activity. As a result, its consequence was excluded. Fifty percent alcohol was selected as a solvent because it had the highest solubility of the oil.

As illustrated in Fig. 4, the extensive different of clear zone was found between $1 \%$ and $2 \%$ lemongrass essential oil application. Mold growth covered $100 \%$ of the disc with $1 \%$ lemongrass essential oil, while the fabric discs with $2 \%-25 \%$ could totally inhibit mold growth. Two percent lemongrass essential oil was the MIC of TK fabric in this research.

Clear zone of both agents was obviously different. Only one concentration (1\%) of lemongrass essential oil generated no clear zone. By contrast, all concentrations of $\mathrm{BKC}$ produced a clear zone. This would be the variation of mass transfer between lemongrass essential oil and BKC [17]. Regarding to its density, vapor (lemongrass essential oil) is much lighter than liquid (BKC). Intermolecular force is another key point of this different. Vapor has a weaker intermolecular force than liquid. Accordingly, vapor could shift much freely. The vapor then transfers to all surface areas in the petri dish, while liquid could only diffuse around the fabric. As a result, the clear zone of lemongrass essential oil covered up the entire surface of media. But the clear zone of BKC depends on diffusion. Our results agreed with those reported by Yousef that vapor phase of lemongrass essential oil could inhibit growth and sporulation of Aspergillus [18]. 


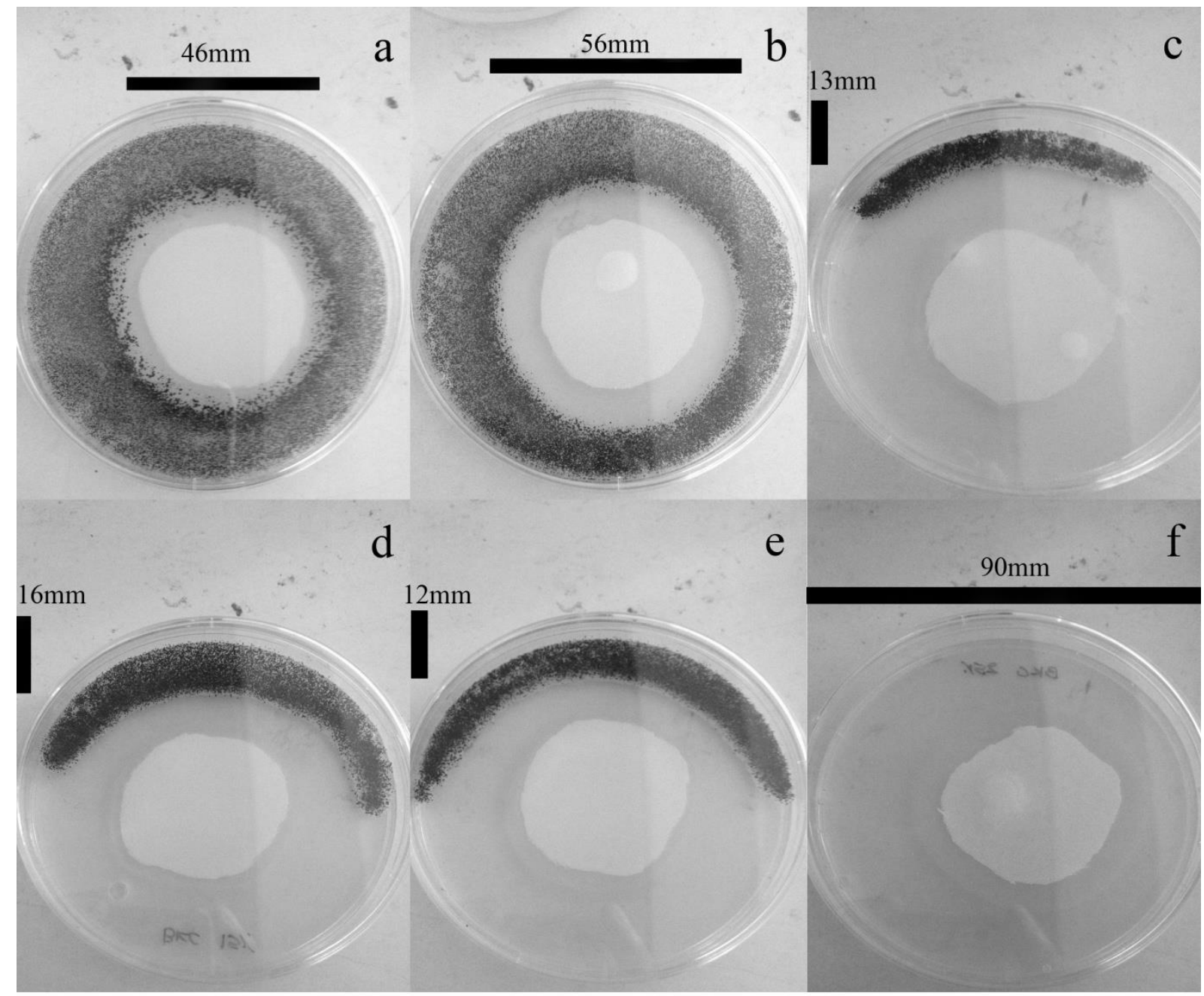

Fig. 1. Observed fungal growth on TK fabric; with BKC concentrations: (a) $1 \% \mathrm{BKC}$; (b) $5 \% \mathrm{BKC}$; (c) 10\% BKC; (d) $15 \%$ BKC; (e) 20\% BKC; (f) 25\% BKC.

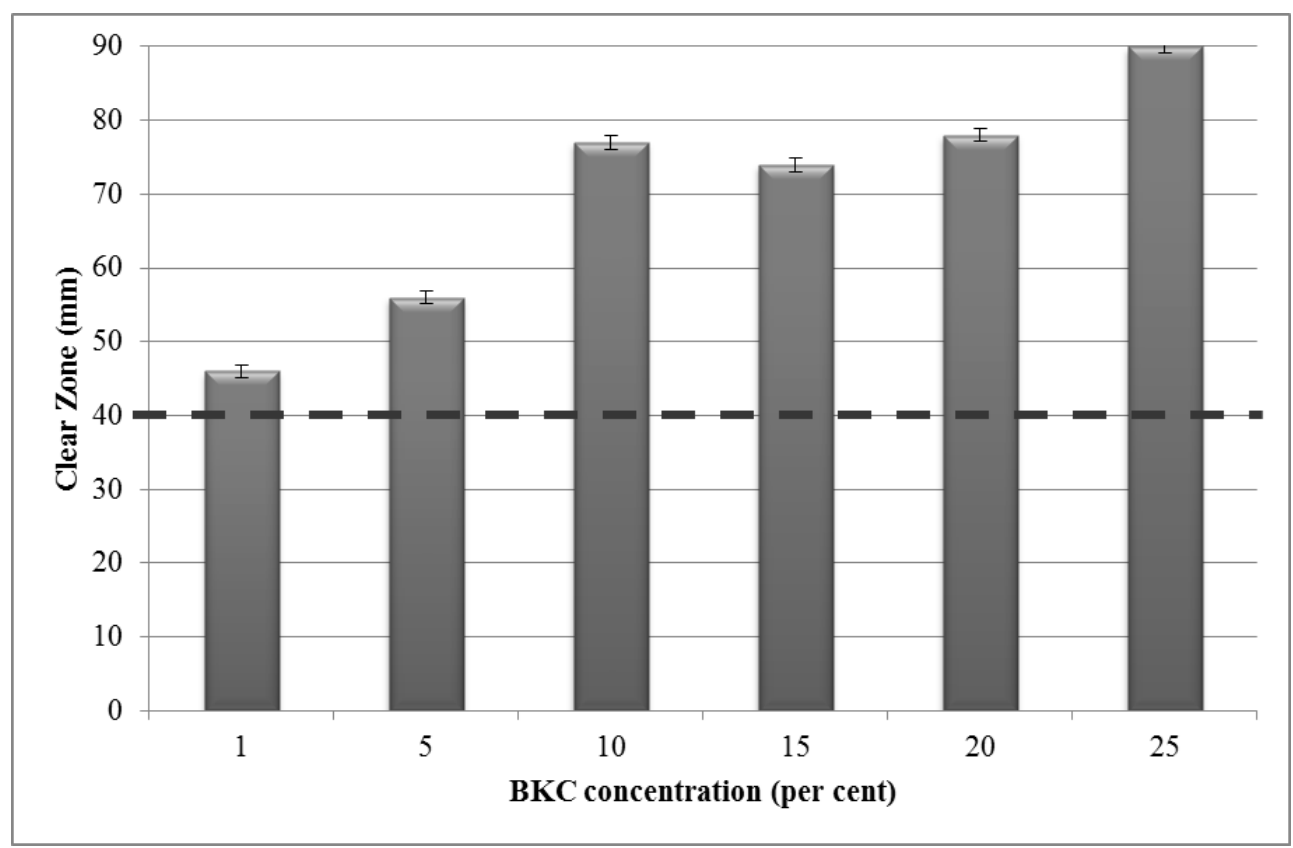

Fig. 2. Correlation between clear zone of fungal growth on TK fabric and BKC concentrations (dashed line at $40 \mathrm{~mm}$ is the size of the fabric discs and is the minimum clear zone in this study). 


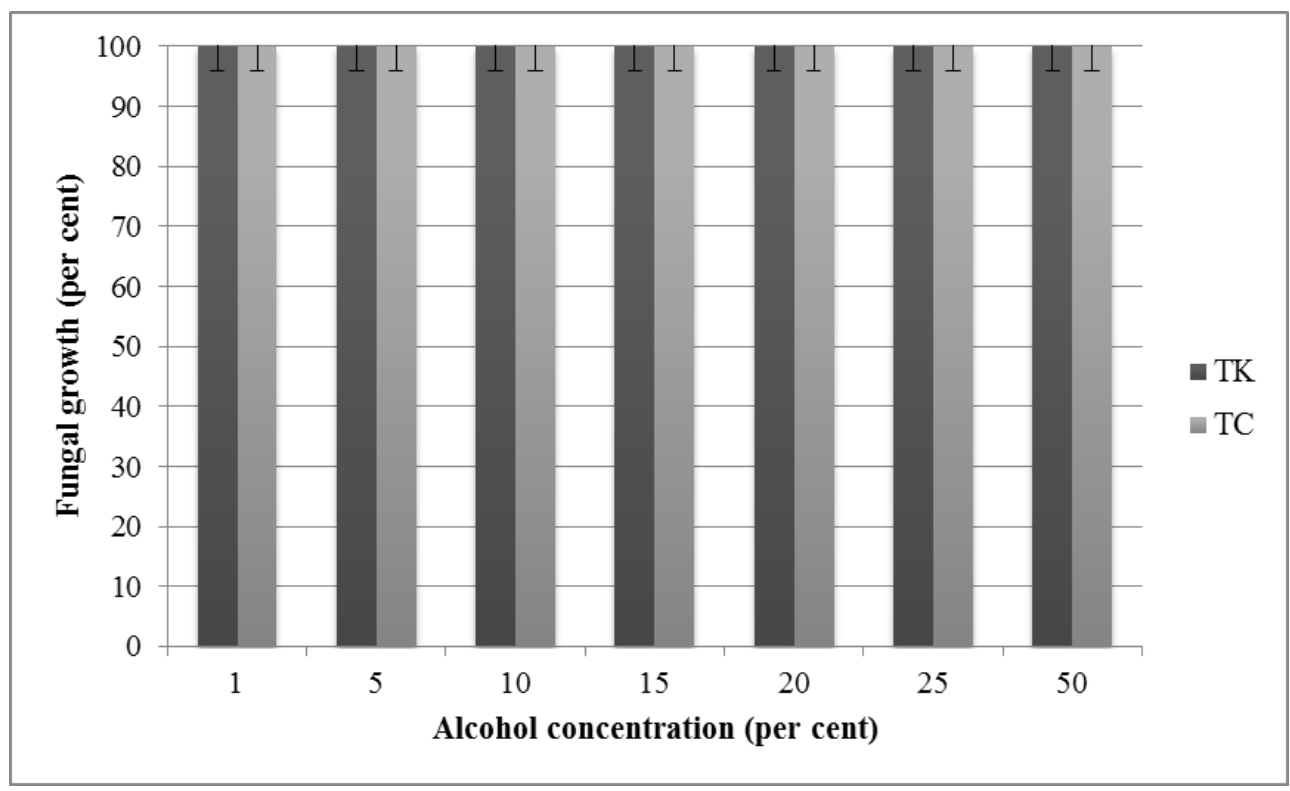

Fig. 3. Correlation between fungal growth on TK/TC fabric and alcohol concentrations.

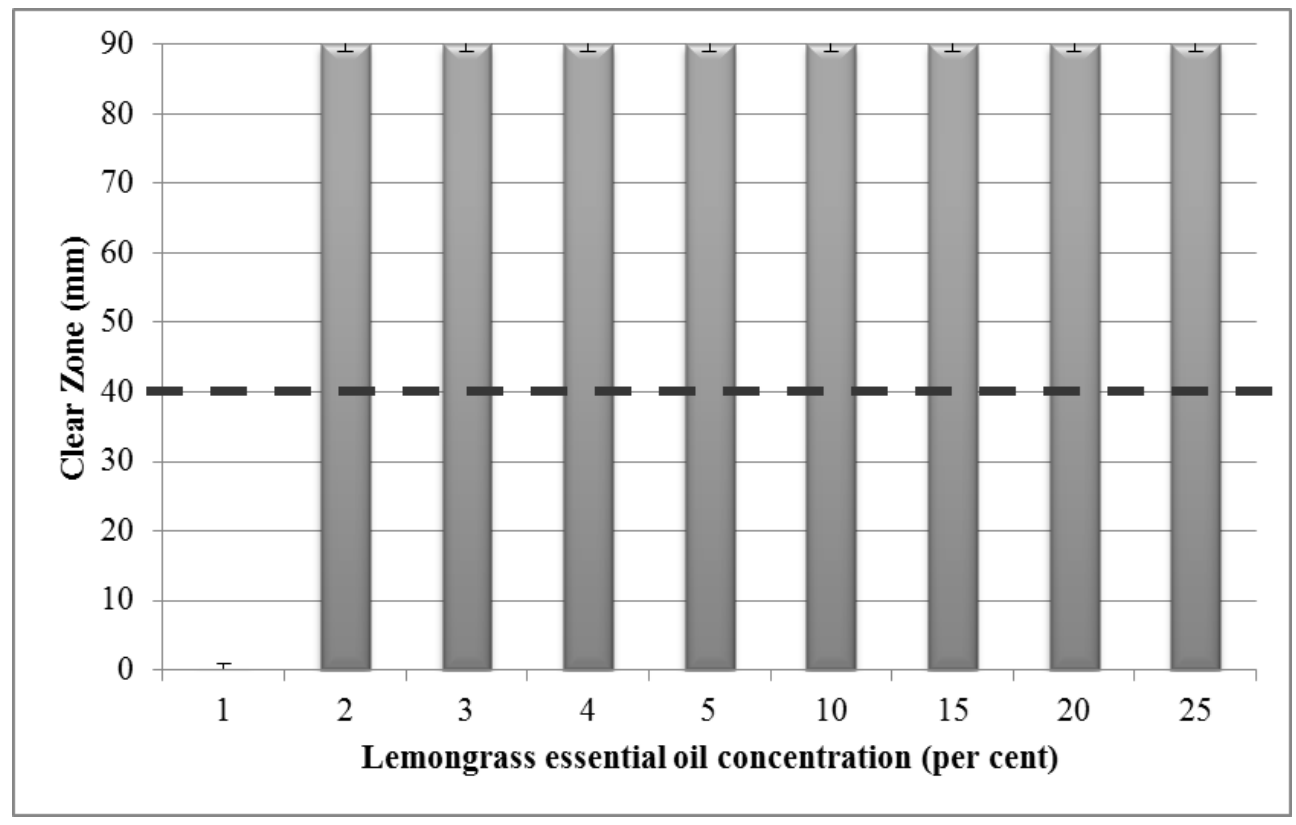

Fig. 4. Correlation between clear zone of fungal growth on TK fabric and lemongrass essential oil concentrations (dashed line at $40 \mathrm{~mm}$ is the size of the fabric discs and is the minimum clear zone in this study).

There was a different efficiency in $5 \%, 10 \%, 15 \%$, and $20 \%$ of both agents on TK fabric. Although BKC has lower efficiency than lemongrass essential oil, both agents could inhibit fungal growth (no growth was observed on the discs). In practical use, an MIC is of interest. One percent $\mathrm{BKC}$ and $2 \%$ lemongrass essential oil were suitable fungicides. When considered the cost of the fungicides as shown in Table 1, 1\% BKC has about 55 times lower cost than $2 \%$ lemongrass essential oil. As the results, $1 \%$ BKC would be recommended for fungal inhibition on TK fabric in this study.
Table 1. Cost of $1 \% \mathrm{BKC}$ and $2 \%$ lemongrass essential oil.

\begin{tabular}{lc}
\hline \multicolumn{1}{c}{ Antifungal agent } & $\begin{array}{c}\text { Cost (baht per } \\
\text { millilitre) }\end{array}$ \\
\hline $1 \%$ BKC & 0.0014 \\
\hline $\begin{array}{l}\% \% \text { lemongrass essential } \\
\text { oil }\end{array}$ & 0.0764 \\
\hline
\end{tabular}

3.2.2. Efficiency on fungal inhibition of $\mathrm{BKC}$ and lemongrass essential oil on TC fabric

The results of $\mathrm{BKC}$ on $\mathrm{TC}$ fabric were similar to those of BKC on TK fabric. TC fabrics with BKC concentrations had a significant difference in clear zone of 
fungal growth. All BKC concentrations determined were larger than $40 \mathrm{~mm}$, except that of the $1 \% \mathrm{BKC}$. As illustrated in Fig. 5, BKC concentrations of 5\%, 10\%, $15 \%, 20 \%$, and $25 \%$ had a $52,58,61,63$, and $64 \mathrm{~mm}$ of clear zones, respectively. However, $1 \% \mathrm{BKC}$ has mold growth on the disc. As a result, $5 \% \mathrm{BKC}$ was the MIC of TC fabric in this study.

Effect of the lemongrass oil on TC fabric was determined using 1\%-10\% of the lemongrass oil in 50\% ethanol. The lemongrass essential oil of $2 \%$ and higher totally inhibit growth of $A$. niger on TC fabric as illustrated in Fig. 6, all lemongrass essential oil concentration except $1 \%$ concentration could inhibit fungal growth. Two percent lemongrass essential oil was the MIC of TC fabric in this study. Interestingly, up to $100 \mathrm{ppm}(0.01 \%)$ of lemongrass essential oil was reported to induce conidia germination of $A$. niger [14]. Tzortzakis and Economakis report could be inferred that at below inhibition concentration the oil could stimulate growth of fungi and caused damage to the product intend to protect.

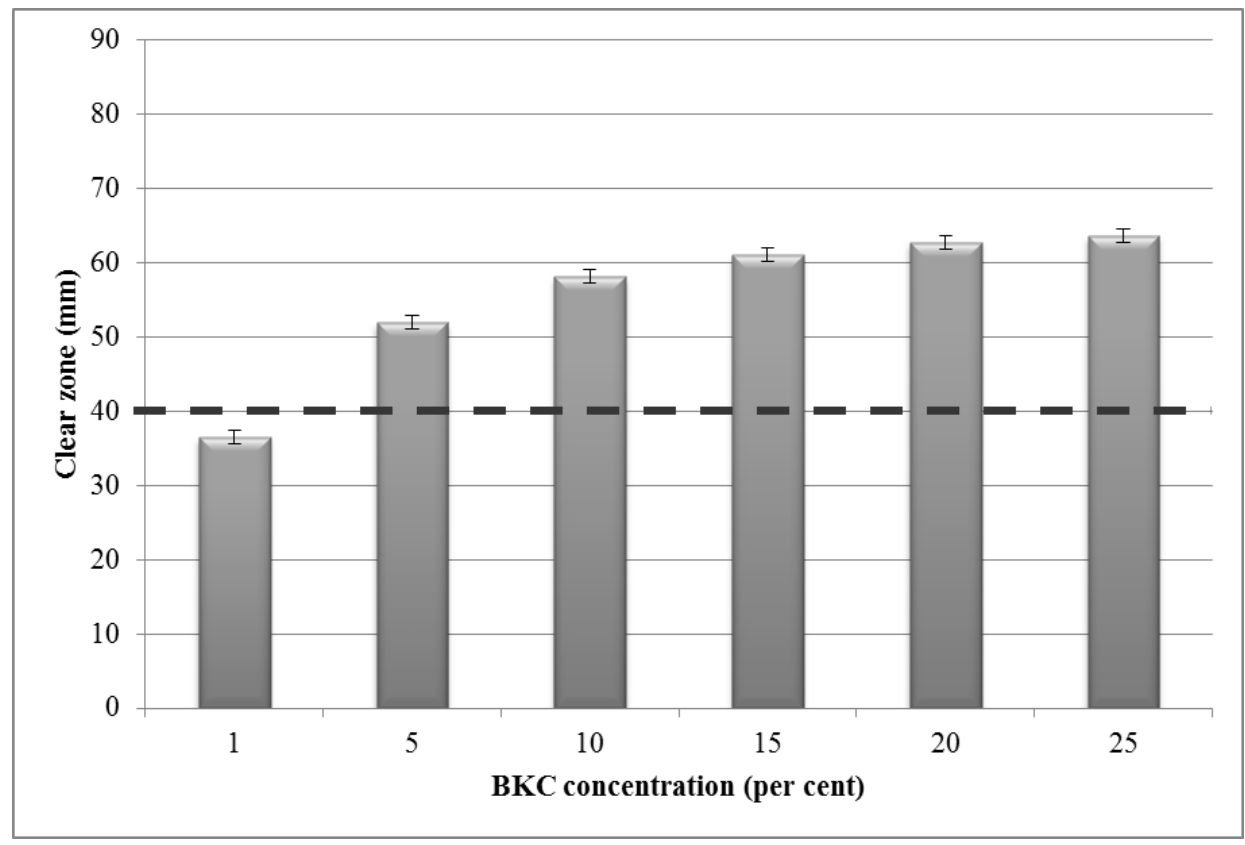

Fig. 5. Correlation between clear zone of fungal growth on TC fabric and BKC concentrations (dashed line at $40 \mathrm{~mm}$ is the size of the fabric discs and is the minimum clear zone in this study).

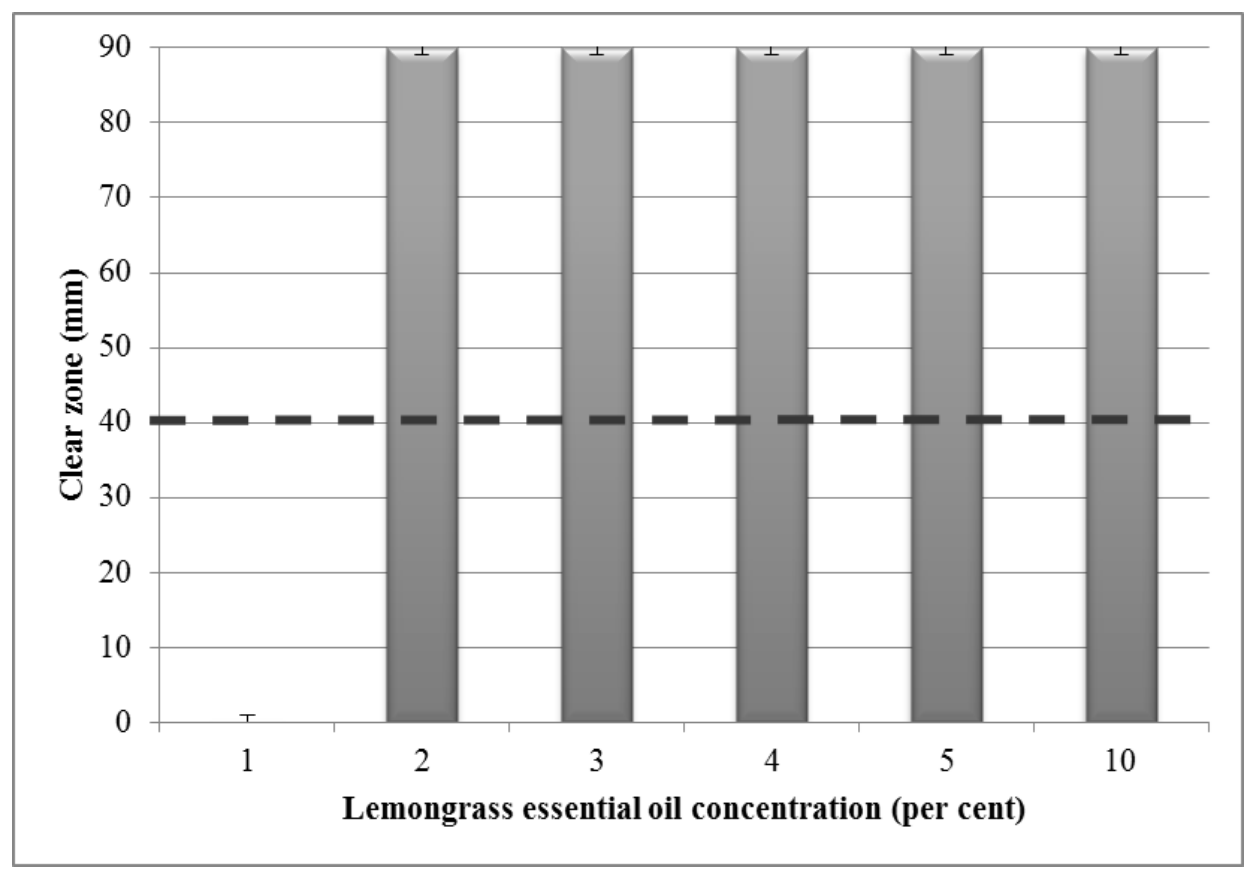

Fig. 6. Correlation between clear zone of fungal growth on TC fabric and Lemongrass essential oil concentrations (dashed line was a specimen size; $40 \mathrm{~mm}$ that was the standard clear zone in this study). 
One percent concentration of both fungicides could not inhibit fungal growth on TC fabric that was different from those of TK fabric. In fungal inhibition on TC fabric, at $5 \%$ and $10 \%$ concentration, lemongrass essential oil is more effective than BKC. But both fungicides could inhibit the fungal growth on the discs as well. In practical use, MIC of $A$. niger on TC fabric was $5 \%$ for $\mathrm{BKC}$ and $2 \%$ for lemongrass essential oil. As illustrated in Table 2 , $5 \% \mathrm{BKC}$ has a lower cost than that of $2 \%$ lemongrass essential oil by about 11 times. This indicated that $5 \%$ $\mathrm{BKC}$ would be recommended for fungal inhibition on TC fabric in this study.

There was a different MIC of BKC between TK and TC fabric. One percent BKC on TK fabric could inhibit the fungal growth but not on TC fabric. The reason of this difference could be from components of fabric. TK is composed of $100 \%$ synthetic fiber that does not absorb moisture. On the other hand, TC is composed of $65 \%$ synthetic fiber and 35\% cotton. Water containment ratios of cellulose and polyester determined by centrifugal dehydration method were $143 \%$ and $50 \%$, respectively [19]. TC fabric absorbs the media solution more than TK, when placed on the media. Cellulose in TC fabric also served as a carbon source of $A$. niger. As a result, to inhibit the fungal growth on $\mathrm{TC}$ fabric, higher $\mathrm{BKC}$ concentration needed to be applied.
Table 2. Cost of 5\% BKC and 2\% lemongrass essential oil.

\begin{tabular}{lc}
\hline \multicolumn{1}{c}{ Antifungal agent } & $\begin{array}{c}\text { Cost (baht per } \\
\text { milliliter) }\end{array}$ \\
\hline $5 \% \mathrm{BKC}$ & 0.0070 \\
\hline $\begin{array}{l}\text { 2\% lemongrass essential } \\
\text { oil }\end{array}$ & 0.0764 \\
\hline
\end{tabular}

\subsection{Effective Duration of Suitable Fungicide}

\subsubsection{Effective duration of $1 \% \mathrm{BKC}$ on $\mathrm{TK}$ fabric}

To determine the frequency of fungicide application to protect against mold growth on TK fabric, prolong incubation with interval inspection was performed. Results from the above experiments showed that $1 \% \mathrm{BKC}$ was a suitable fungicide for fungal inhibition on TK fabric. One percent $\mathrm{BKC}$ was selected to test for the effective duration of BKC on TK fabric as shown in Fig. 7. Fungal growth on the fabric was not observed after 7 and 15 days of incubation (Fig. 7(a) and Fig. 7(b)) but fungal growth was observed on TK fabric on day 19 and beyond (Fig. 7(c) and Fig. 7(d)). From the experiment, the effective duration of $1 \%$ BKC on TK fabric was 18 days.

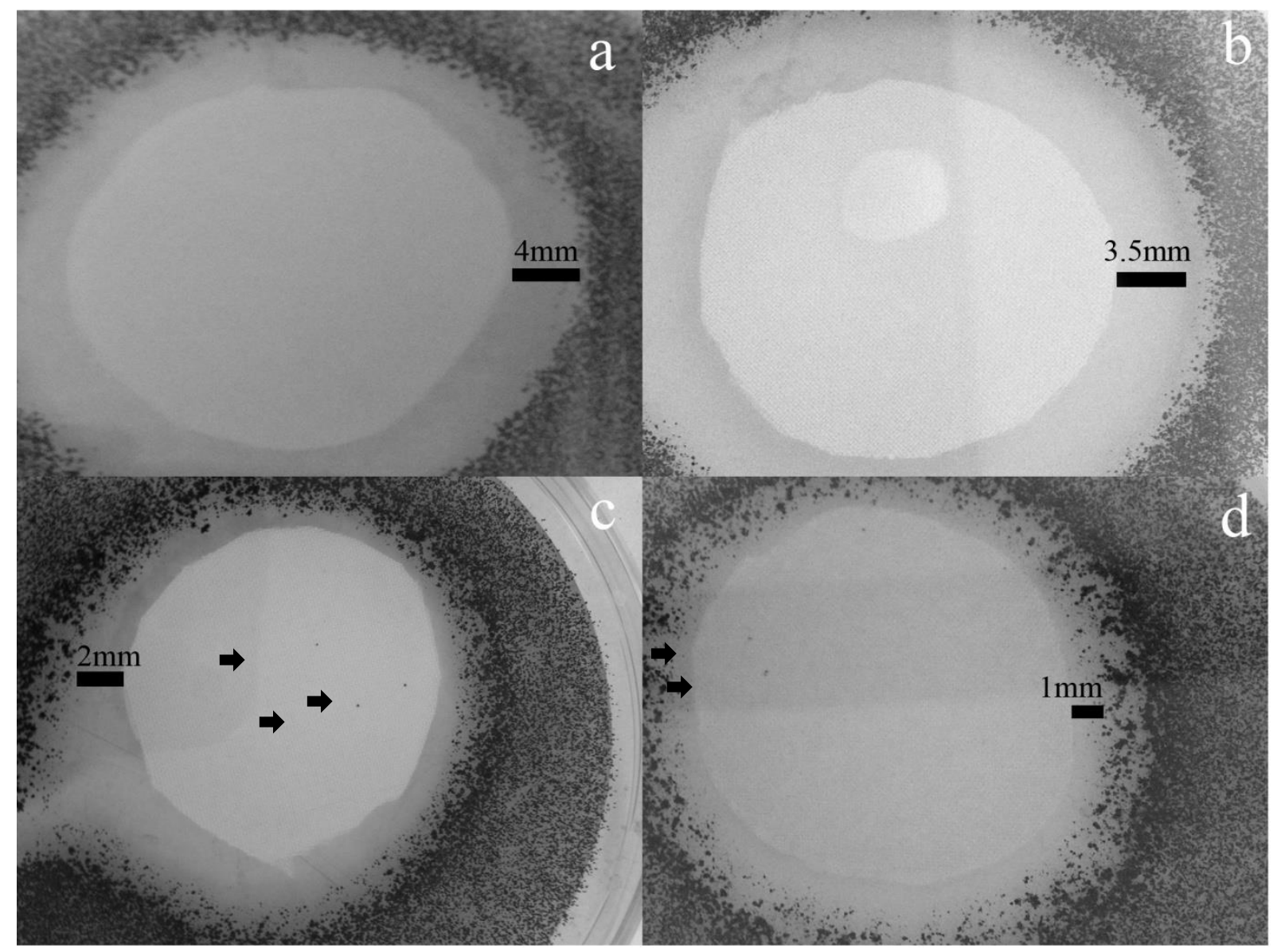

Fig. 7. Fungal growth on TK fabric with $1 \%$ BKC at various duration: (a) 7 days; (b) 15 days; (c) 19 days; (d) 30 days. Arrows indicate fungal growth on the fabric. 


\subsubsection{Effective duration of $5 \% \mathrm{BKC}$ on TC fabric}

To determine the frequency of fungicide application to protect against mold growth on TC fabric, prolong incubation with interval inspection was performed. Results from the experiment in 3.1.2. showed that $5 \%$ $\mathrm{BKC}$ was a suitable fungicide for fungal inhibition on TC fabric. Five percent BKC was selected to test for the effective duration of BKC on TC fabric as shown in Fig. 8. Fungal growth on the fabric was not observed after 30 days of incubation, although inhibition zone was reduced over time course of incubation. From the experiment, the effective duration of $5 \% \mathrm{BKC}$ on TC fabric was at least 30 days.

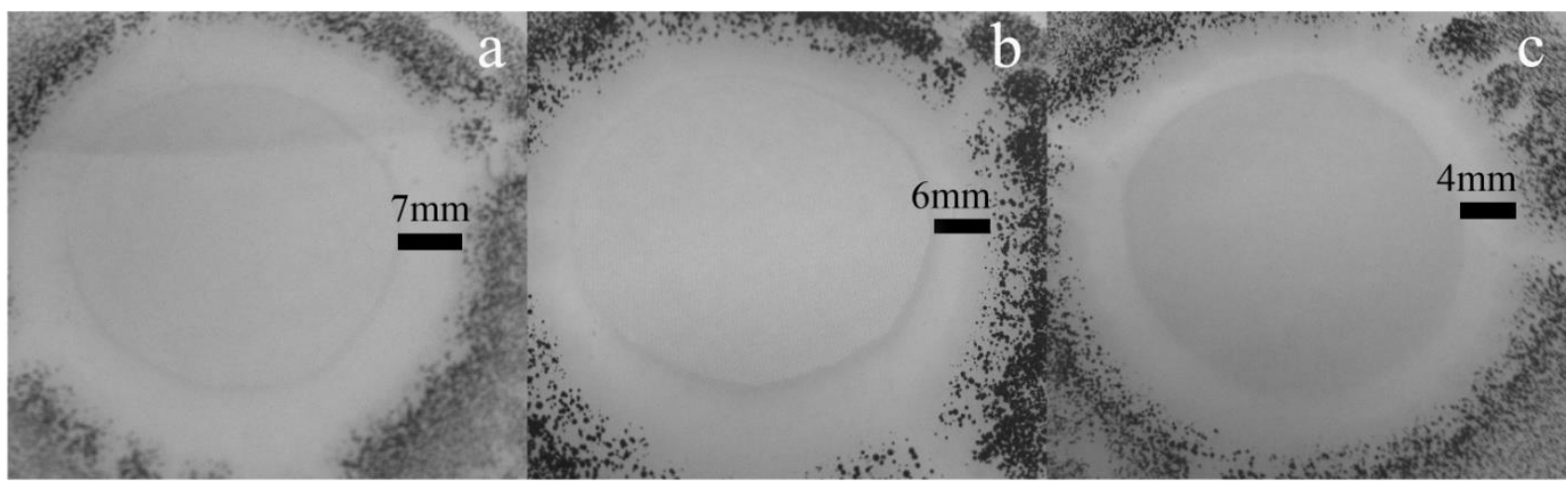

Fig. 8. Fungal growth on TC fabric with 5\%BKC at various duration: (a) 7 days; (b) 15 days; (c) 30 days.

\subsection{Damage of Polyester Fabric Caused by Fungicides}

In this study $\mathrm{BKC}$ and lemongrass essential oil were used to treat polyester fabric. Distilled water and 50\% ethanol were used as diluent controls for both fungicides, respectively. Prolong exposure to certain chemicals had been reported to damage yarn or discolor the fabric [20]. In order to determine whether these chemicals deteriorate the TK and TC fabric, deionized water (DI water), $1 \%$, $5 \%, 10 \%, 15 \%, 20 \%$, and $25 \%$ BKC, $1 \%$ lemongrass essential oil, and 50\% ethanol were tested on both types of fabrics. Eighty percent BKC, and 2\% - 100\% lemongrass essential oil could not be weighed because the fabrics were attached on petri dish as shown in Fig. 9. The damage of polyester fabric was analyzed by the loss of specimen weight and characteristic of fiber, including yarn arrangement and yarn appearance. Before the treatment, each fabric was weigh then treated with the chemicals or diluents then air dried and kept for 3, 7, 10, and 14 days.
At each time point, the fabrics were weighed. As shown in Table 5, weights of all treated samples were stable after 3 days as a result of diluents evaporation. This suggested that, fabrics were not damaged by the fungicides.

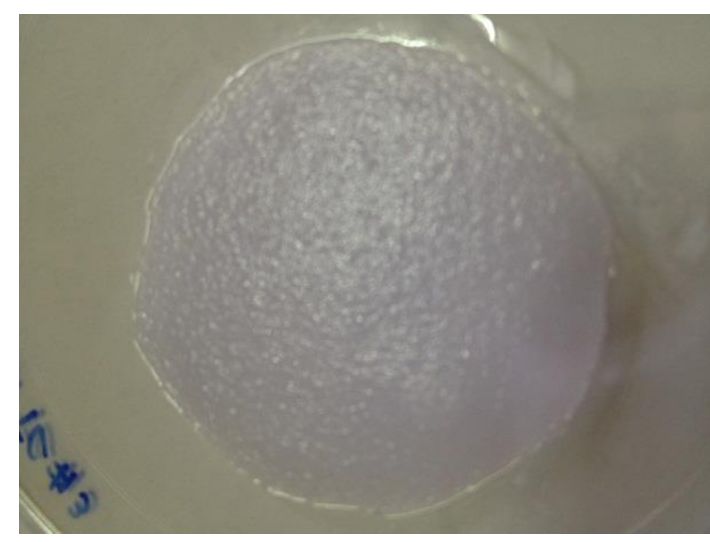

Fig. 9. Fabric with fungicide was attached on petri dish.

Table 5. The weight loss of TK and TC fabric at various durations compared with day 1.

\begin{tabular}{lcccc}
\hline \multirow{2}{*}{ Chemical } & \multicolumn{2}{c}{ The weight loss of TK/TC compared with day 1 (\%) } \\
\cline { 2 - 5 } & Day 3 & Day 7 & Day 10 & Day 14 \\
\hline DI water & $56.97 / 44.09$ & $57.02 / 45.62$ & $57.02 / 47.61$ & $57.02 / 47.71$ \\
\hline 50\% ethanol & $67.13 / 45.81$ & $67.19 / 48.45$ & $67.19 / 50.43$ & $67.19 / 50.53$ \\
\hline BKC 1\% & $55.27 / 42.81$ & $55.32 / 43.66$ & $55.49 / 45.60$ & $55.49 / 45.70$ \\
\hline BKC 5\% & $35.35 / 26.43$ & $40.20 / 39.69$ & $41.28 / 42.41$ & $42.40 / 42.70$ \\
\hline BKC 10\% & $34.90 / 26.06$ & $38.91 / 36.95$ & $38.91 / 40.49$ & $39.66 / 42.30$ \\
\hline BKC 15\% & $20.39 / 16.67$ & $28.80 / 31.75$ & $31.65 / 36.54$ & $34.03 / 40.13$ \\
\hline BKC 20\% & $20.52 / 17.86$ & $29.29 / 25.60$ & $33.14 / 32.16$ & $36.61 / 37.44$ \\
\hline BKC 25\% & $18.99 / 15.08$ & $28.39 / 27.81$ & $32.75 / 35.59$ & $38.17 / 43.52$ \\
\hline lemongrass Oil 1\% & $50.76 / 34.95$ & $50.94 / 35.57$ & $51.00 / 35.73$ & $51.00 / 35.85$ \\
\hline
\end{tabular}


Damage to the yarn after treatment with fungicides was determined under microscope. TK fabric treatment with 1-25\% BKC and 1\% lemongrass essential oil did not affect yarn arrangement and integrity after 14 days post treatment as shown in Fig. 11 comparing to the yarn treated on day 1 in Fig.10. Yarn characteristics of TC fabric treated similarly as TK baric also showed no damage when inspected under microscope (data not shown).

TK and TC fabric were not damaged by $1 \%, 5 \%, 10 \%$, $15 \%, 20 \%, 25 \%$ BKC and 1\% lemongrass essential oil. The lost weight of fabric caused by evaporation; was not by fabric damage. In addition, fiber characteristic was not changed over time. This suggested that $1 \%, 5 \%, 10 \%$, $15 \%, 20 \%, 25 \%$ BKC and 1\% lemongrass essential oil did not damage TK and TC fabric in this study.

\section{Conclusions}

$\mathrm{BKC}$ and lemongrass essential oil are shown to be effective fungicides. Although $\mathrm{BKC}$ would be required at higher concentrations than those of lemongrass essential oil (based on 100\% efficiency), in practical use, an MIC could be proposed. MIC on TK and TC fabric of BKC was 1 and 5\%, respectively, while MIC on TK and TC fabric of lemongrass essential oil was $2 \%$. The $2 \%$ lemongrass essential oil has higher cost than $1 \%$ and $5 \%$ $\mathrm{BKC}$ at about 55 and 11 times, respectively. Regarding to reasonable expense, $1 \%$ and $5 \% \mathrm{BKC}$ would be a suitable agent in this study. TK and TC fabric should be sprayed every 18 and 30 days with $1 \%$ and $5 \%$ BKC, respectively. Moreover, both fungicides did not cause damage to yarn of the fabric. Lemongrass essential oil evaporates quickly, and the scent of lemongrass essential oil may be deemed unacceptable by some individuals [21-22]. As a result, application of Lemongrass essential oil on fabrics for a long-term storage in a close container is an environmental friendly method to prevent mold growth on fabrics.

\section{Acknowledgements}

This work has been financially supported by Graduate school of Chulalongkorn University and Research Unit: Air Quality Control Management, Chulalongkorn University, Thailand.

The instrument in this study was supported by department of microbiology, faculty of science, Chulalongkorn University.

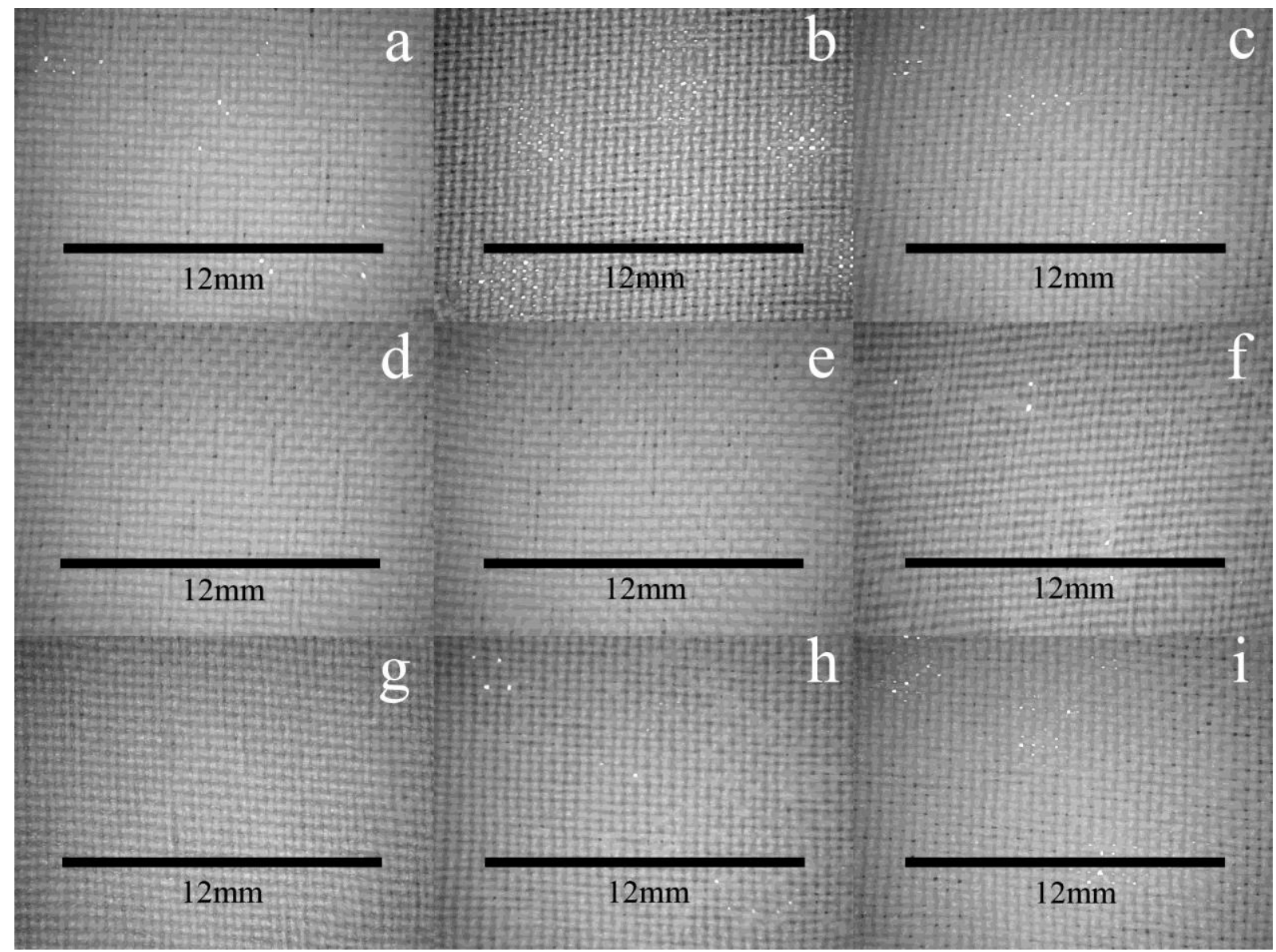

Fig. 10. TK Fiber with chemicals day 1: (a) DI water; (b) 50\%alcohol; (c) $1 \% \mathrm{BKC}$; (d) 5\%BKC; (e) $10 \% \mathrm{BKC}$; (f) 15\%BKC; (g) 20\%BKC; (h) 25\%BKC; (i) $1 \%$ lemongrass essential oil. 


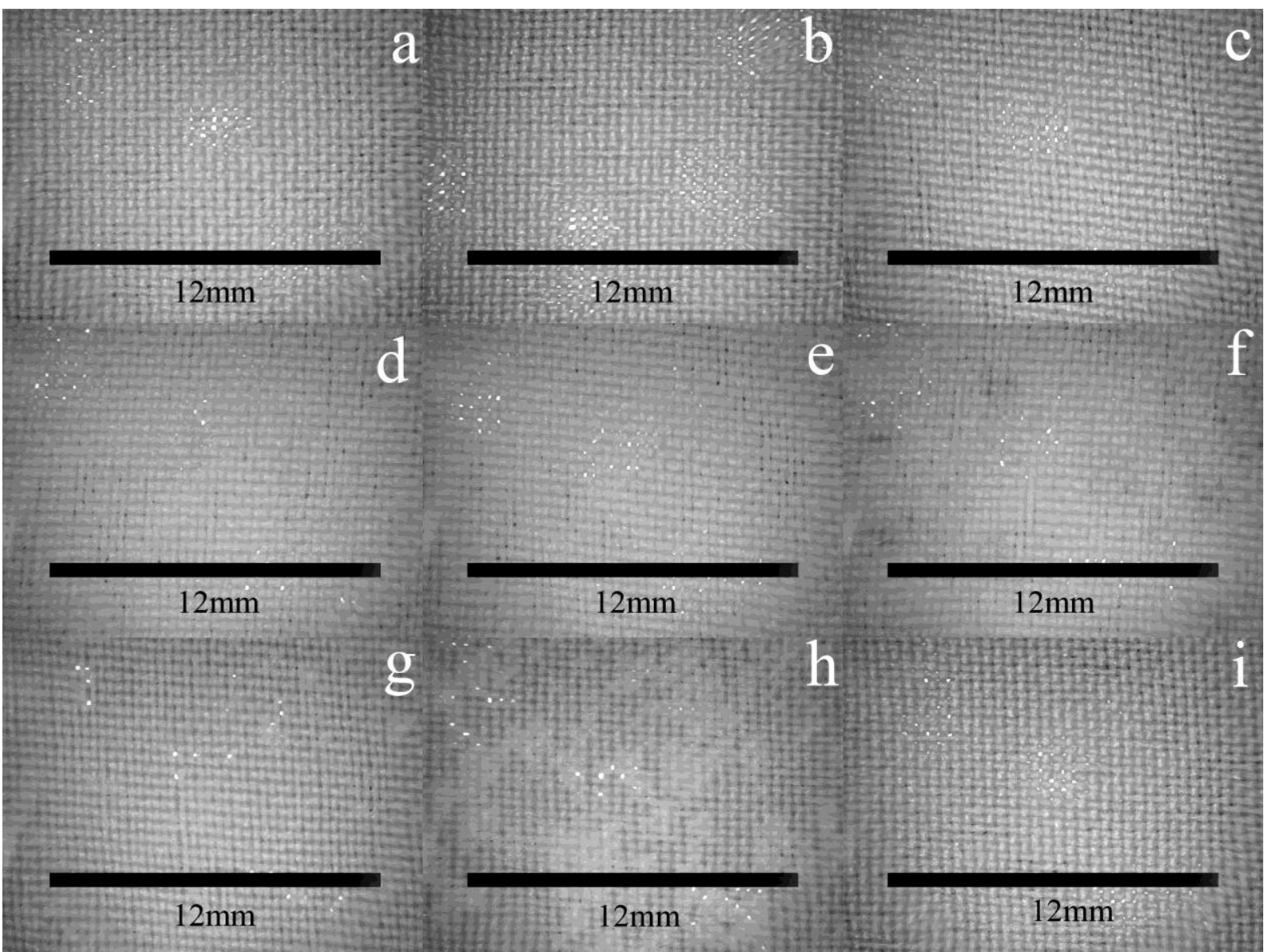

Fig. 11. TK Fiber with chemicals on day 3: (a) DI water; (b) 50\%alcohol; (c) $1 \% \mathrm{BKC}$; (d) $5 \% \mathrm{BKC}$; (e) $10 \% \mathrm{BKC}$; (f) 15\%BKC; (g) 20\%BKC; (h) 25\%BKC; (i) 1\% lemongrass essential oil.

\section{References}

[1] A. M. Lewinska, R. H. Peuhkuri, C. Rode, B. Andersen, and J. B. Hoof, "Rapid detection and identification of Stachybotrys and Chaetomium species using tissue PCR analysis" Journal of Microbiological Methods, vol. 130, pp. 115-122, 2016. Available:

https://doi.org/10.1016/j.mimet.2016.09.005

[2] S. N. Baxi, J. M. Portnoy, D. Larenas-Linnemann, W. Phipatanakul, C. Barnes, S. Baxi, C. Grimes, W. E. Horner, K. Kennedy, D. Larenas-Linnemann, and E. Levetin, "Exposure and health effects of fungi on humans," The Journal of Allergy and Clinical Immunology: In Practice, vol. 4, no. 3, pp. 396-404, 2016.

[3] S. Fernandez-Rodriguez, R. Tormo, J. M. Maya, I. Silva, and A. Gonzalo, "Outdoor airborne fungi captured by viable and non-viable methods," Fungal Ecology, vol. 7, pp. 16-26, 2014.

[4] N. Hamada and T. Fujita, "Effect of air-conditioner on fungal contamination," Atmospheric Environmental, vol. 36, pp. 5443-5448, 2002. Available: https://doi.org/10.1016/S1352-2310(02)00661-1

[5] M. Ljaljević-grbić, J. Vukojević, and M. Stupar, "Fungal colonization of air-conditioning systems," Archives of Biological Sciences, vol. 60, no. 2, pp. 201-206, 2008.
[6] R. K. Bush, J. M. Portnoy, A. Saxon, A. I. Terr, and R. A. Wood, "The medicine effects of mold exposure," The Journal of Allergy and Clinical Immunology, vol. 117, no. 2, pp. 326-332, 2006.

[7] Z. Filip, "Polyurethane as the sole nutrient source for Aspergillus niger and Cladosporium herbarum, European Journal of Applied Microbiology and Biotechnology, vol. 7, pp. 277-280, 1979.

[8] W. C. Zhu, J. Gyamfi, L. N. Niu, G. J. Schoeffel, S. Y. Liu, F. Santarcangelo, S. Khan, K. C. Y. Tay, D.H. Pashley, and F. R. Tay, "Anatomy of sodium hypochlorite accidents involving facial ecchymosis-A review," Journal of Dentistry, vol. 41, pp. 935-948, 2013.

[9] K. R. Seddon, "Ionic liquids for clean technology," Journal of Chemical Technology and Biotechnology, vol. 68, pp. 351-356, 1997.

[10] M. S. Fortunato, S. Baroni, A. J. González, J. D. Á. Roncancio, A. Storino, C. Parise, A. Gallego, and S. E. Korol, "Biodegradation and detoxification of benzalkonium chloride in synthetic and industrial effluents in upflow biofilm aerobic reactors," Water, Air, \& Soil Pollution, vol. 230, no. 4, p. 79, 2019.

[11] M. M. Reynolds, K. E. Greenwood-Quaintance, R. Patel, and J. S. Pulido, "Selected antimicrobial activity of topical ophthalmic anesthetics, Trans. Vis. 
Sci. Tech., vol. 5, no. 4, p. 2, 2016. doi: 10.1167/tvst.5.4.2.

[12] Y. Xu, Y. He, X. H. Li, C. W. Gao, L. T. Zhou, S. T. Sun, and G. G. Pang, "Antifungal effect of ophthalmic preservatives phenylmercuric nitrate and benzalkonium chloride on ocular pathogenic filamentous fungi," Diagnostic Microbiology and Infectious Disease, vol. 75, no. 1, pp. 1-4, 2012.

[13] M. N. Boukhatem, M. A. Ferhat, A. Kameli, F. Saidi, and H. T. Kebir, "Lemon grass (Cymbopogon citratus) essential oil as a potent anti-inflammatory and antifungal drugs," Libyan Journal of Medicine, vol. 9, no. 1, p. 25431, 2014. doi:10.3402/ljm.v9.25431

[14] N. G. Tzortzakis and C. D. Economakis, "Antifungal activity of lemongrass (Cympopogon citratus L.) essential oil against key postharvest pathogens," Innovative Food Science and Emerging Technologies, vol. 8, pp. 253-258, 2007.

[15] American Association of Textile Chemists and Colorists, AATCC Technical Manual. Research Triangle Park, North Carolina, USA, 2010.

[16] F. R. Passamani, T. Hernandes, N. A. Lopes, S. C. Bastos, W. D. Santiago, M. D. Cardoso, and L. R. Batisa, "Effect of temperature, water activity, and $\mathrm{pH}$ on growth and production of ochratoxin A by Aspergillus niger and Aspergillus carbonarius from Brazilian grapes," Journal of Food Protection, vol. 77, 1947-1952, 2014. doi: 10.4315/0362-028X. JFP-13495
[17] J. R. Welty, C. E. Wicks, R. E. Wilson, and R. E. Rorrer, Fundamentals of Momentum, Heat, and Mass Transfer. New York, USA: Hamilton Printing, 2008.

[18] S. A. Al Yousef, "Antifungal activity of volatiles from lemongrass (Cymbopogon citratus) and peppermint (Mentha piperita) oils against some respiratory pathogenic species of Aspergillus," Int J Curr Microbiol App Sci, vol. 2, no. 6, pp. 261-72, 2013.

[19] O. Wada and Y. Takatera, "Physical properties and wear test of water absorbent polyester fibers," Journal of the Textile Machinery Society of Japan, vol. 30, no. 4, pp. $191-99, \quad 198 . \quad$ Available: https://doi.org/10.4188/jte1955.30.91

[20] T. Topalovic, V. A. Nierstrasz, L. Bautista, D. Jocic, A. Navarro, and M. M. Warmoeskerken, "Analysis of the effects of catalytic bleaching on cotton," Cellulose, vol. 14, no. 4, pp. 385-400, 2007.

[21] P. Martins, P. Sbaite, C. Benites, and M. Wolf Maciel, "Thermal characterization of orange, lemongrass, and basil essential oils," Chemical Engineering Transactions, vol. 24, pp. 463-468, 2011.

[22] A. Hadjilouka, M. Polychronopoulou, S. Paramithiotis, P. Tzamalis, and E. Drosinos, "Effect of lemongrass essential oil vapors on microbial dynamics and listeria monocytogenes survival on rocket and melon stored under different packaging conditions and temperatures," Microorganisms, vol. 3, no. 3, pp. 535-550, 2015. doi:10.3390/microorganisms3030535

Thanyanuch Kriangkraipipat, photograph and biography not available at the time of publication.

Nattawat Wittayakunsathit, photograph and biography not available at the time of publication.

Thawat Ngamsritrakul, photograph and biography not available at the time of publication.

Sirima Panyamethekul, photograph and biography not available at the time of publication. 